\title{
Developing Educational Mobile Application Architecture using SOA
}

\author{
Z. Al-Khanjari, Z. Al-Kindi, E. Al-Kindi and N. Kraiem \\ Department of Computer Science, College of Science, Sultan Qaboos University \\ PO Box 36, Al-Khoud 123, Muscat, Sultanate Oman \\ zuhoor@squ.edu.om,\{m109871;m109107\}@student.squ.edu.om, \\ naoufel@squ.edu.om
}

\begin{abstract}
Mobile technologies have enormous potential to transform ordinary institutions content to electronic content. This change contributes to advance the level of performance of these institutions, whether educational or services institutions. Therefore, developing such applications using one of the well-known Software Engineering paradigms is important to support the institutions. Most of educational organizations in Oman don't provide a clear mobile application architecture that supports different organizational services. The significant purposes of these services are to provide an environment with more malleable procedures, satisfying student's needs, and achieving a quick access to information. Moreover, this paper proposes an extension tool to support students in all educational fields to send their appeal against exams results via a mobile application that integrates one of the well-known software architectures called Service-Oriented Architecture (SOA). In this paper, Service-Oriented Architecture for post result mobile application is used to integrate a set of services that have been implemented in a web server with the existing post result application.
\end{abstract}

Keywords: Post Result, Service Oriented Architecture (SOA), Mobile Application, HTTP, J2EE, JSON

\section{Introduction}

Providing a social and an educational mobile phone application is the intention of almost all developers [1-3]. Mobile applications consist of a numerous number of software that runs on a mobile device and performs definite tasks for the users. Mobile application is a recent and a rapid developing segment of the global information and communication technology, friendly, inexpensive, downloadable and implemented in most of the mobile phones. Moreover, developing such application to support educational fields is beneficial. Introducing mobile application technologies with internet has successfully enabled educational institutions to provide their students and staff members with various online educational services [4]. With the current developments in mobile technology, further possibilities are emerging to come up with such services via mobile devices. By extending the educational services to wireless medium, the educational institutions can probably bring great convenience to those off-campus learners who do not always have time to find internet enabled with computers to get the important information from their academic institutions. This paper presents an ongoing research project which proposes the development of a post result mobile application for students in different education organization e.g. colleges and universities. This electronic tool will let the students to send their appeal request against their exam result via their mobile phone. In addition, appeal service follows a special service architecture called Service-Oriented Architecture. SOA defines a set of key concepts of software architecture and their relations, where a service can be integrated and elaborated with other components by 
following a model of publishing services and applications and their universal access [5-7]. It is a software design and software architecture design pattern based on discrete portions of software providing application functionality as services to other applications [8]. Indeed, publishing these services "appeal against exam result" in different educational institutions is advantageous for both students and lecturers since it reduces the time consumed to do the same operation manually. Therefore, this paper focuses on providing more details about appeal service with a context for a deeper understanding of services and Service-Oriented Architectures for software solutions. The paper is structured in five sections. It included related work, mobile application and SOA, case study for post results mobile application using SOA and the conclusion.

\section{Related Work}

Mobile applications exposed new markets and infinitely extending the reach of businesses. There is a worldwide affirmative influence of using mobile application. The usage of mobile application in industrialized nation introducing key abilities to faculties and people, societies of developing countries are invigorating themselves and making a new type of IT arrangement. There are several mobile applications that use ServiceOriented Architecture to implementing their application services. Therefore, the main objective of this paper is to develop post result mobile application using SOA. Before providing the details, it is worth to go via the previous studies to find out the main concepts behind this idea.

In 2005, Chen and Kinshuk described a prototype for a mobile system of educational services [9]. The prototype showed how mobile education service system can be a useful complement to the current desktop systems. Both the students and the educators will benefit from the mobile education system for convenient and instant access to education assets. Moreover, Pramsane and Sanjaya presented a development of education services based on Short Message Service (SMS) [10]. The university as an educational organization has a great deal with the information that can be provided to the students, such as grade release and enrollment information. Shahriza and colleagues studied the utilization of mobile phone services in the educational environment, explored the nature of mobile phone use among university students and investigated the perception of university students on mobile phone uses in library and information services [11]. Therefore, a high majority of the respondents indicated that there is an increasing need of mobile application and $\mathrm{m}$-services in different sectors. Some previous researches presented these m-services which can be implemented using SOA. For example, SánchezNielsen and colleagues discussed the importance of m-services that used SOA and showed how this architecture in widely used in web services [12]. However, the researchers proposed a service oriented architecture that addresses the different issues for instance, dynamical integration of new services by providers at any time, dynamical discovery of available services and the use of open source software to develop the solution. Moreover, Herrington and Herrington presented the usage of mobile technologies in higher education implications for future theory development [8]. Natchetoi and colleagues investigated a novel lightweight mobile SOA-based architecture for business applications running on J2ME enabled devices e.g. cell Phones. The paper introduced various techniques based on SOA for mobile devices [13]. In addition, Daniel and Younas provided a literature which cussed on the challenges and problems which might be faced during engineering of web application. Also, in this direction highlighted the solution the research community worked on [14]. Regarding the security in mobile applications, Payne discussed that there are significant challenges to building secure mobile applications. However, he mentioned about the availability of ways to reduce these risks. Here, the author discussed a few of the best practices associated with secure mobile development [15]. Also, Lin and colleagues conferred high-quality articles 
describing security algorithms, protocols, policies, and frameworks for applications running on modern mobile platforms for example android, iOS, and Windows phone [16]. As mobile applications grow, the researchers believe that mobile application security will continue to be a rich research field. In order to implement these mobile applications for the purpose of their current paper, the developers need to combine SOA with secure components. Genevra and colleagues showed that SOA is a software design and software architecture design pattern based on discrete portions of software providing application functionality as services to other applications [17].

\section{Mobile Application and SOA}

There are different ways for improving the functionality and developing a mobile application services. One of these influential approaches is SOA. While the SOA concept which is used by many developers during their implementation phase of their systems, SOA vary from existing distributed technologies in providing scalability and reusability mechanism for developing different services. Furthermore, SOA with a universal establishment of values has the enhanced reusability of existing resources or investments in the enterprise and allowed different business to create applications that can be built on top of new and existing applications.

\subsection{Implementing Mobile Application Using SOA}

Extending the benefits of mobile phone use is now the focus for countless development projects. Although many technical projects target assisting aid workers or providing group tools for whole communities, mobile phones have uniquely become easily accessible and useful for individual users. SOA have turn out to be one of the most common architecture as a way to support the business practices of an organization. Moreover, SOA is built around services on a network that interconnect with each other. The services are loosely coupled, have well-defined, liberated interfaces, and are sustainable. Furthermost business applications oblige performing significant amounts of data processing, either locally or across high-speed networks. However, currently most of current cell phones cannot satisfy these requirements. The main difficulties that limit the development of business applications on mobile devices remain to be unreliable network performance and limited data storage. Therefore, using SOA as an approach will provide web service invocation, data exchange, transformation, and interfacing with the user. SOA is a structural model that contains a set of components such as web services technology that can be used and integrated with other components. SOA is defined as "an application architecture with which all functions are defined as independent services with well-defined invokable interfaces that can be called in defined sequence to form business process" [18]. The service will act as the core object in developing architecture. It provides an interface where other components can interact and communicate with its capabilities over networks using standard protocols. In mobile application, SOA is a back-end access that involves a set of standards to exchange data. SOA can be implemented via web services including different format for instance XML. Web service is a software application specified by a unique URI that can be used through its interface. The service interface is encapsulated by Web Services Definition Languages (WSDL). By the help of XML, WSDL will offer a description via network services that allows SOA components to be used. Afterwards, it is published in the Universal Discovery Description Integration (UDDI) which is known as Broker or Registry. Next, the service can be transferred through message passing over HTTP [19]. The basic architecture of SOA consists of three main components: 


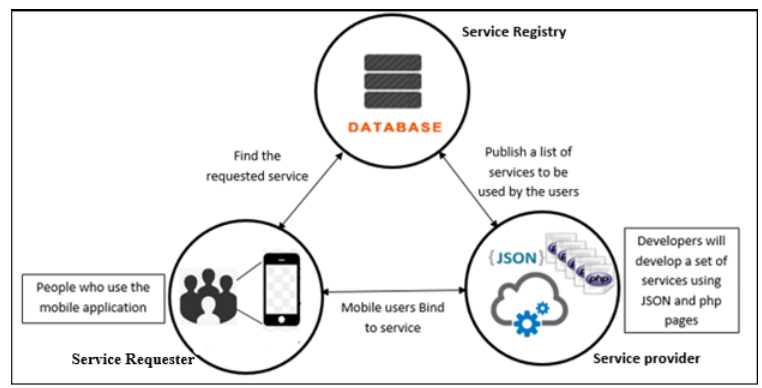

Figure 1. Three Roles in Service-Oriented Architecture (SOA)

The first component is namely a Service Provider which is responsible for designing and implementing services, specifying the interface to these services and publishing information about these services in a central registry (directory service). The second component is called Service Requester it is also called client or entity that requires certain functions (services) to perform some tasks or it may be an application or service that relies on interaction with a boot or some services. The third one is a Service Registry (Broker) which is a location of service description (i.e. where the Service Provider publishes a description of the service) [20]. The components mentioned above interact with each other as given below:

- Publish - records a description of the service in the directory of services, covering the registration of its capabilities, interface, performance and quality that it offers.

- Find - searches for services registered in the directory of services, provided that they meet the desired criteria and it can be used in a process of business, taking into account the description of the published service.

- Bind - this operation relies on the service requested or boots an interaction with the service at runtime using the information obtained in discovery of the service.

\subsection{Secure Mobile Application with SOA}

A large-scale and a comprehensive SOA for secure mobile applications and transactions was intended. Safeguarding an open architecture, similar to SOA, can prove to be much more challenging than securing a closed system in traditional software development [21]. There numerous challenges for instance:

- Comprehensive: many security services are provided by the architecture.

- Scalable: provide the possibility for interlinking mutually independent deployments.

- Modular: new services, functions and components can easily be added to the architecture.

- Expandable: mobile applications can easily be linked to the architecture and can be utilized its services.

- Open meaning that integration of new components is based on utilization of standard-based web services and interfaces.

Based on this secure mobile applying using SOA, functions of all mobile applications are exposed to mobile users as services. These services are easy and flexible to use for end-users in the way that user can access services anytime, anywhere, and through various communication channels. It is also accessible and flexible for service providers to manage their mobile services. 


\section{Case study: Post Result Mobile Application Using SOA}

The developers of the current research decided to develop post result mobile application using SOA for students. This application is an educational tool that is used to support the students during their studies. This system provides appeal service which is a new service that can be accessed using android devices. Students can send their appeal request during the appeal duration and waiting for the results. This enables the students to check the appeal results. At its most basic, the proposed approach as services aimed to implement educational mobile application (Post Result Mobile Application) using SOA concept. The two services will use the concept of SOA in terms of implementing the services in remote access server and receiving the students' data. System requires the students to use their institution id and password in order to access the application. The application is not a replacement for any certain existing systems. Moreover, the current developers follow SOA as services where they use some services provided by providers. In order to make this application more flexible, the developers connected the apps to remote access where all students' details have been stored. Although, the application developed using J2EE platform which is the dominant development platform for SOA applications, SOA is not by any means limited to this platform. Platforms such as J2EE does not only deliver the framework for developers to naturally participate in the SOA, but also, by their essential nature, bring a mature and proven infrastructure for scalability, reliability, availability, and performance to the SOA world. Newer specifications such as Java API for XML Binding (JAXB), used for mapping XML documents to Java classes, Java API for XML Registry (JAXR), used for interacting with the UDDI registries in a standard manner, and Java API for XML-based Remote Procedure Call (XML-RPC), used for invoking remote services in J2EE 1.4 facilitate the development and deployment of Web services that are portable across standard J2EE containers, while simultaneously interoperating with services across other platforms such as .Net.

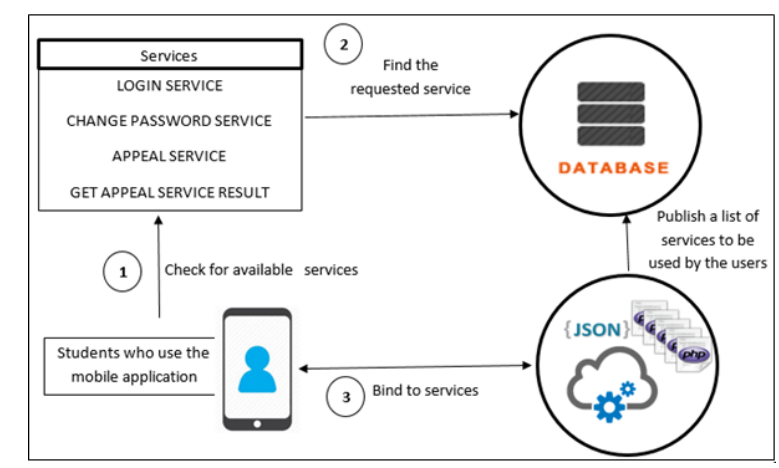

Figure 2. The Overall Architecture of the Proposed Approach

Figure 2 demonstrates the framework of post result mobile application that integrates different components using SOA architecture. The figure illustrates the services that system provides to students, including login services and change password as authentication module. Moreover, Figure 2 shows appeal forms and appeal result as appeal operation module. All services can use the database that stores all students' related information. Besides, once the student has the right to access the application then he/she will be able to use the other services. Also, Figure 2 illustrates the request across the network to access the services via JSON (JavaScript Object Notation) which is a lightweight data-interchange presentation. It is based on a subset of the JavaScript Programming Language to respond to the client with accurate data. 


\subsection{Developing Post Result Mobile Application Using SOA Concept}

Based on the above framework, researchers can apply SOA to be used for building new post result mobile application using services provided by service providers. These services will be in form of JSON data received from remote server. So, the new system will be composed of independent services supplied by the providers.

Therefore, the requirement for developing new service:

* Server: create services and publish them for public use.

* Client: access for requesting a desired service required to build a system.

* Services: implemented functionalities that will be created by a server.

* Database: store services those are created by a server.

\subsection{Secure Post Result Mobile Application with SOA}

The proposed system introduces a special service to secure the application and the data provided by the system. The application used serval services within architecture that implemented as a separate security layer within a set of infrastructure services. Moreover, these services will be accessing via URL which in supported by service provider. "A service is a mechanism to enable access to one or more capabilities, where the access is provided using a prescribed interface and is exercised consistent with constrains and policies as specified by the service description" [22]. In the current system, the developers provide an interface that materialized the authorize user while login to the application. Furthermore, by using of SOA concept the application implemented a service that sends an email when the user login to the system. This property of the service will eliminate the threat or decline the vulnerability. In addition, these services will be implementing using web service technology. Web service will provide authentication, confidentiality, and a reasonable level of message integrity between the application users and the main services that are provided by the service provider. In the extended communications framework of SOA, there is no direct access from the users to the service. The only way is the users can interact via a well-defined interface to access the services. This is a strong point to protect the services and the data of the application.

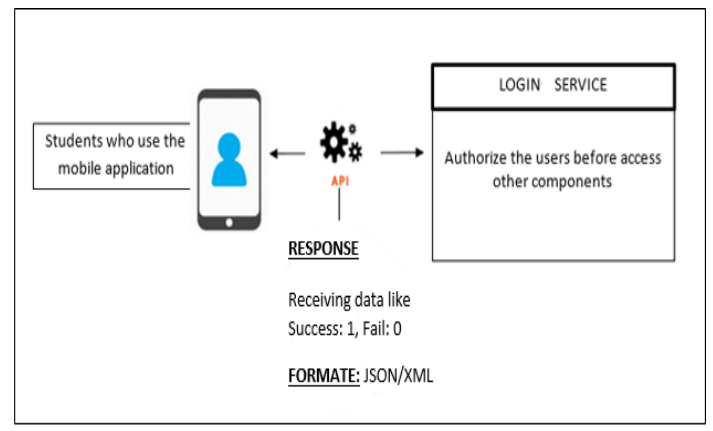

Figure 3. Using the Service API and Login Service to Secure the Proposed System

\section{Conclusion and Future Work}

This paper proposes an educational approach that supports mobile phone application. The proposed approach uses SOA to provide flexible functionalities for the system. This paper claims that neither similar tool using SOA nor were services available in the literature. Therefore, the best alternative is to develop that service as software then publish it in the cloud and integrate it with the new system. The researchers established 
login service, change password service and appeal service with the use of SOA. In future, developers will distribute the admin service in internal repository to be available on the same mobile app. Moreover, this service will contain update appeal request, delete appeal request and other services. In addition the researchers will expend the idea to cover more other mobile platforms for instance, IOS platform.

\section{References}

[1] Z. Al-Khanjari, "Developing a Common Personalization Framework for the E-Application Software System", Journal of Emerging Technologies in Web Intelligence (JETWI), Special Issue on Novel Techniques and Applications of Data Analytics, Academy Publisher, vol. 5, no. 2, (2013), pp. 188-195.

[2] Y. Jamoussi, Z. Al-Khanjari and N. Kraiem, "A Framework to Evaluate E-learning Based on Social Networking", International Journal of Current Research and Academic Review (IJCRAR), vol. 2, no. 4, (2014), pp. 26-42.

[3] Z. Al-Khanjari, K. Al-Kindi, A. Al-Zidi and Y. Baghdadi, "M-Learning: The New Horizon of Learning in SQU", Journal of Engineering Research (TJER), SQU, vol. 11, no. 2, (2014), pp. 14-25.

[4] A. Al-Zeidi, K. Al-Kindi and Z. Al-Khanjari, "SQU Future: From E-Learning to M-Learning Application Development", Proceedings of the International Information Systems Conference (IISC), College of Commerce, Sultan Qaboos University, Oman, (2011).

[5] Z. Al-Khanjari and Y. Al-Roshdi, "Extending the Functionality of LMS to Support Computer Science Education Using Plug-in Tools", Journal of Emerging Technologies in Web Intelligence (JETWI), Academy Publisher, vol. 6, no. 2, (2014), pp. 220-225.

[6] Z. Al-Khanjari, Y. Al-Roshdi and N. Kraiem, "Virt-Res: Developing Extended Architectural Design for Computer Science Virtual Resources Using SO", International Journal of Software Engineering and its Applications (IJSEIA), Academy Publisher, vol. 8, no. 9, (2014), pp. 125-136.

[7] Z. Al-Khanjari and Y. Al-Roshdi, "Developing Virtual Lab to support the Computer Science Education in Moodle", The International Conference on Remote Engineering and Virtual Instrumentation (REV2015), Bangkok, Thailand, February 25-28, (2015).

[8] A. Herrington and J. Herrington, "Authentic mobile learning in higher education", International Educational Research Conference, Fremantle, Western Australia, (2007).

[9] J. Chen and D. Kinshuk, "Mobile technology in educational services", Journal of Educational Multimedia and Hypermedia, vol. 14, no. 1, (2005), pp. 91.

[10] S. Pramsane and R. Sanjaya, "Mobile education services based on SMS and their architecture comparison", In Proceedings of the Third International Conference on eLearning for Knowledge-based Society, Bangkok, Thailand, vol. 49, no. 1, August 3-4, (2006), pp. 2.

[11] N. S. A. Karim, S. H. Darus and R. Hussin, "Mobile phone applications in academic library services: a students' feedback survey”, Campus-Wide Information Systems, vol. 23, no. 1, (2006), pp. 35-51.

[12] E. S. Nielsen, S. M. Ruiz and J. R.Pedrianes, "An open and dynamical service oriented architecture for supporting mobile services", In Proceedings of the international conference on Web engineering, ACM.USA, (2006), pp. 121-128.

[13] Y. Natchetoi, V. Kaufman and A. Shapiro, "Service-oriented architecture for mobile applications", In Proceedings of the 1st international workshop on Software architectures and mobility, ACM, (2008), pp. 27-32.

[14] F. Daniel and M. Younas, "Mobile web and service applications: Guest editors' introduction", Service Oriented Computing and Applications, vol. 6, no. 4, (2012), pp. 337-339.

[15] J. Payne, "Secure mobile application development", IT Professional, vol. 15, no. 3, (2013), pp. 6-9.

[16] Y. Lin, C. Huang, M. Wright and G. Kambourakis, "Mobile application security", Computer, vol. 47, no. 6, (2014), pp. 21-23.

[17] E. C. Genevra, O. Patrick and O. F. Eleonu, "Service oriented architecture: The future of information technology”, Academic Research International, vol. 5, no. 4, (2014), pp. 1.

[18] Z. Qinghua, D. Bo, T. Feng and C. Wei, "A service-oriented approach to integration of e-learning Information and Resource Management Systems", Proceedings of $12^{\text {th }}$ International Conference on Computer Supported Cooperative Work in Design (CSCWD), Xi'an, China, April 16-18, (2008).

[19] A. Brown, S. Johnston and K. Kelly, "Using service-oriented architecture and component-based development to build web service applications", Rational Software Corporation, (2002).

[20] J. Fonseca, Z. Abdelouahab, D. Lopes and S. Labidi, "A security framework for SOA applications in mobile environment”, arXiv preprint arXiv: 1004.0774 (2010), April (2010), pp. 1-18.

[21] D. T. Sanders, J. A. Hamilton Jr and R. A. MacDonald, "Supporting a service-oriented architecture", In Proceedings of the 2008 Spring simulation multi conference, Society for Computer Simulation International, (2008), pp. 325-334.

[22] OASIS Open, "Reference Model for Service-Oriented Architecture 1.0 Committee Specification 1", www.oasisopen. org. Retrieved October 10, 2008, from [online]:http://www.oasis-open. org/committees/download.php/19679/soa-rm-cs.pdf, August 2, (2006). 
International Journal of Multimedia and Ubiquitous Engineering Vol.10, No.9 (2015) 